\title{
Quiz
}

\section{Teaching EEG series 2: Mapping the field of spike potential for source localization}

\author{
G.R.K. Sarma ${ }^{1}$, N. So ${ }^{2}$ \\ ${ }^{1}$ Professor of Neurology, St. John's Medical College Hospital, Bengaluru, Karnataka, India \\ ${ }^{2}$ Clinical Professor, Department of Neurology and Neurophysiology, Washington University Medical Center, Washington, USA
}

\section{Questions:}

1. Map the field of the spike activity seen in Fig. 1.

2. Is the field map biologically plausible?

3. Which is the actual source of this spike?

\section{Answers:}

1. The discharges appear to have a wide field involving the entire left hemisphere. There is an upward deflection in the left frontal region that can indicate negativity at Fp1. But

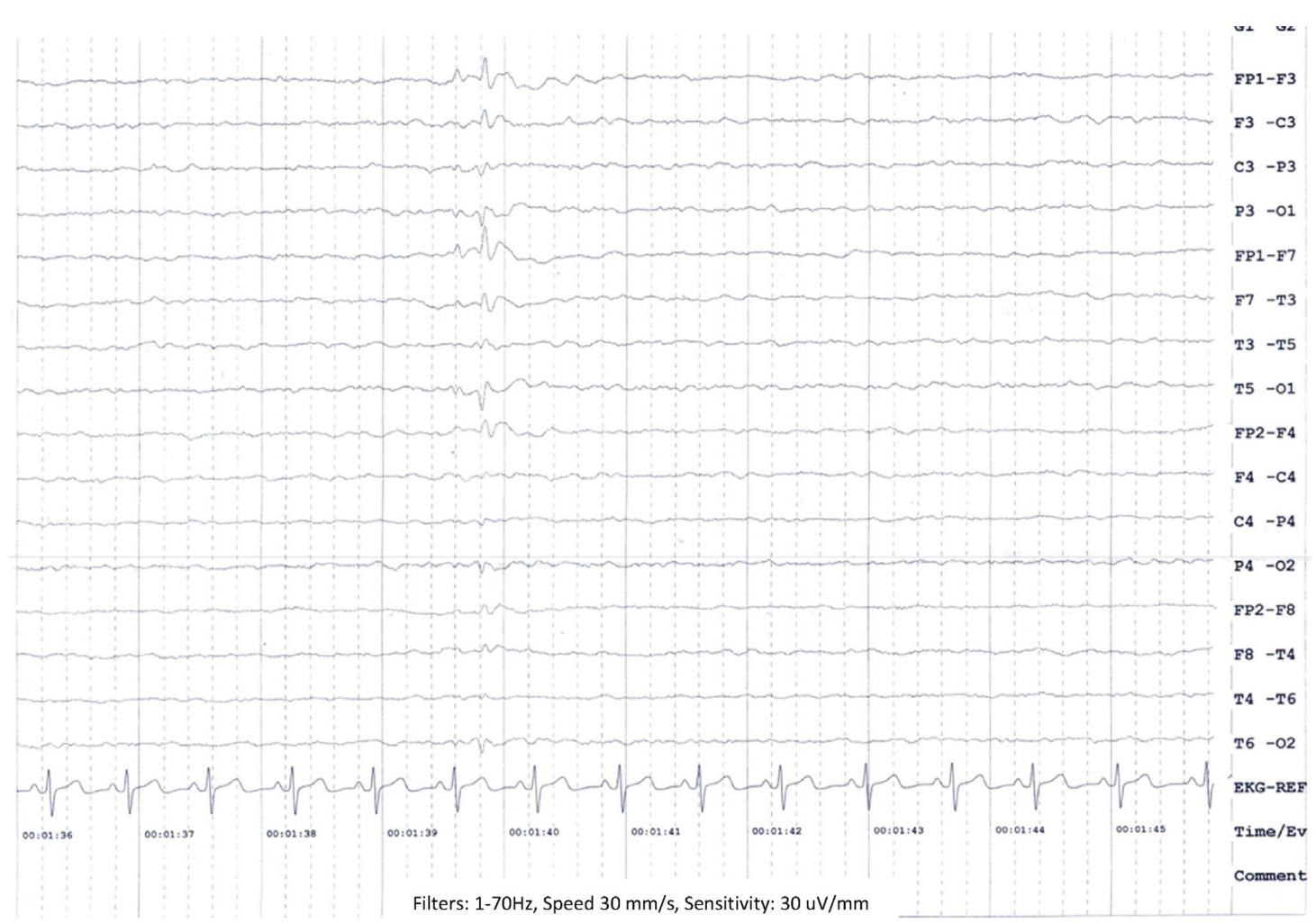

Fig. 1

E-mail address: grk_sarma@yahoo.com. 


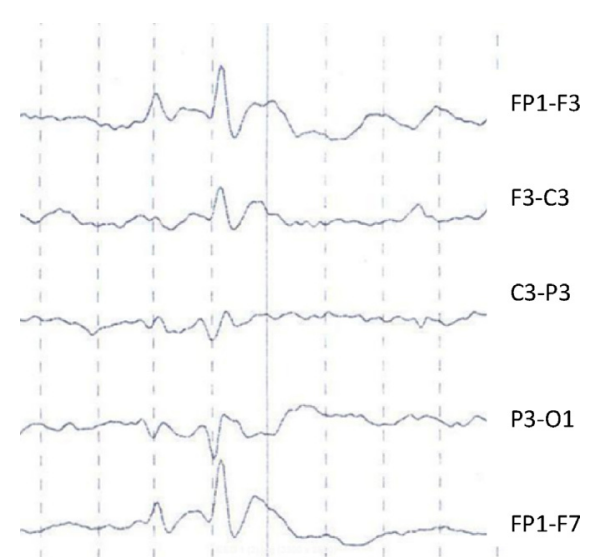

Fig. 2 - The spike activity seen in Fig. 1 has been zoomed in.

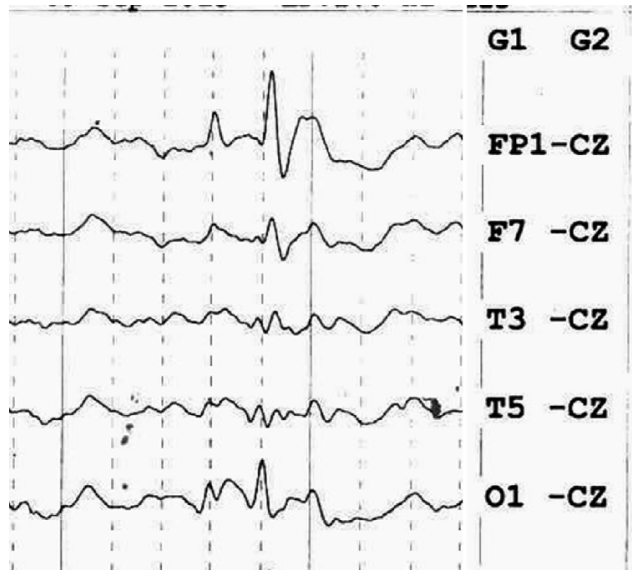

Fig. 3 there is also a downward deflection in the left posterior head region that suggests negativity at 01 . Alternatively, there may be a "positive" divergent phase reversal in the left parasagittal chain at C3, meaning a maximum positivity at $\mathrm{C} 3$ and minimal positivity at Fp1 and $\mathrm{O} 1$.

2. A large central positivity at C3 would be highly unusual for an epileptiform discharge in the absence of a prior craniotomy and cortical resection exposing the inner surface of the cortical layer.

3. On closer inspection (Fig. 2), the divergence at C3 is not truly in phase at the 2 adjacent channels of F3-C3 and C3-P3. Likewise the spike components at Fp1-F3 and P3-O1 are not truly synchronous. Thus, it is not possible to invoke volume conduction from a single source to explain this discharge. The peak occipital negativity at P3-O1 leads the frontal negativity of Fp1-F3 by $30 \mathrm{~ms}$ as can be readily appreciated in the $\mathrm{Cz}$ referential montage (Fig. 3). This indicates that the source of the spike is in the left occipital region with propagation to the left frontal region. This EEG is from a child diagnosed with Panayiotopoulos syndrome and his brain MRI was normal.

\section{Acknowledgements}

We thank Mrs. Elizabeth VT, EEG technician at St. John's Medical College for her assistance and Dr. Sujit Kumar, Consultant Neurologist, Sagar Hospital, Bengaluru for valuable inputs.

\section{Reference}

Yoshinaga H, Koutroumanidis M, Kobayashi K, Shirasawa A, Kikumoto $\mathrm{K}$, Inoue $\mathrm{T}$, Oka $\mathrm{M}$, Ohtsuka Y. EEG dipole characteristics in Panayiotopoulos syndrome. Epilepsia. 2006;47(4):781-7.

Take home message: Accurate mapping of the field potential prevents erroneous conclusions and permits correct localization of the source. Attention to the time of occurrence of the spike in different regions over the scalp permits one to hypothesize on its source and propagation. 